\title{
An open-label, phase 1 study of androgen receptor antagonist, apalutamide in Japanese patients with metastatic castration-resistant prostate cancer
}

\author{
Tomohiro Tsuchiya ${ }^{1} \cdot$ Keiichiro Imanaka $^{2} \cdot$ Yuki Iwaki $^{2} \cdot$ Ryo Oyama $^{2} \cdot$ Katsuyoshi Hashine $^{3} \cdot$ Akito Yamaguchi $^{4}$. \\ Hiroji Uemura ${ }^{5}$
}

Received: 26 March 2019 / Accepted: 30 July 2019 / Published online: 24 August 2019

(c) The Author(s) 2019

\begin{abstract}
Background Apalutamide, a nonsteroidal potent androgen receptor antagonist, was safe and effective in patients with nonmetastatic castration-resistant prostate cancer (nmCRPC) and metastatic-CRPC (mCRPC) in global studies. In this phase 1 study, safety, pharmacokinetics (PK), and efficacy of apalutamide were evaluated in Japanese patients with mCRPC.

Methods In this open-label, multi-center study, patients received apalutamide $240 \mathrm{mg}$ (once-daily, orally) for first 1 week (PK week) during which PK parameters were assessed. 1 week later (Cycle 1 Day 1), after reassessing safety, continuous daily dosing (4 weeks/cycle; once-daily orally) was initiated. Endpoints evaluated were: safety, tolerability, PK and antitumour efficacy of apalutamide. Dose-limiting toxicities (DLTs) were evaluated during PK week and Cycle 1.

Results All six patients received apalutamide. The most common treatment-emergent adverse events (TEAEs) were abdominal discomfort, nasopharyngitis, dysgeusia, rash, and hot flush [2/6 patients (33.3\%) each]. No death or DLTs were reported. Grade 3 TEAEs were spinal-cord compression and renal disorder (1/6 patient each). In continuous daily dosing period, PK steady-state of apalutamide was reached approximately by week 4 . A significant accumulation of apalutamide was observed (mean accumulation index 3.55), based on $\mathrm{AUC}_{0-24}$. Median (range) serum prostate-specific antigen level decreased from $54.42(8.92-310.11) \mathrm{ng} / \mathrm{mL}$ at baseline to $11.70(0.37-47.74) \mathrm{ng} / \mathrm{mL}$ at week 12 with $\geq 50 \%$ reduction in $4 / 6(66.7 \%)$ patients and $90 \%$ reduction in $2 / 6(33.3 \%)$ patients.

Conclusion Apalutamide had manageable safety profile, without any DLT or any new safety signals, and favourable efficacy in Japanese mCRPC patients. Thus, it was ascertained to be an adequate dosage regimen in Japanese mCRPC patients.

Trial registration ClinicalTrials.gov identifier: NCT02162836.
\end{abstract}

Keywords Androgen receptor antagonist $\cdot$ Apalutamide $\cdot$ Metastatic castration-resistant prostate cancer $\cdot$ Prostate cancer

The results of this study were presented at the 56th annual meeting of Japan Society of Clinical Oncology, 18-20th October 2018, Japan.

Electronic supplementary material The online version of this article (https://doi.org/10.1007/s10147-019-01526-7) contains supplementary material, which is available to authorized users.

\footnotetext{
Hiroji Uemura

hu0428@yokohama-cu.ac.jp

Gifu University Hospital, Gifu, Japan

2 Janssen Pharmaceutical K.K., Tokyo, Japan

3 National Hospital Organization Shikoku Cancer Centre, Matsuyama, Japan
}

\section{Introduction}

Prostate cancer is the 6th leading cause of cancer death in men worldwide (307,000 deaths in 2012) [1]. Prostate cancer is estimated as one of the most common type of cancers in Japanese men and the sixth largest cause of cancerrelated death [2-4]. Androgen deprivation therapy remains

\footnotetext{
4 Harasanshin Hospital, Fukuoka, Japan

5 Yokohama City University Medical Centre, 4-57, Urafune-cho, Minami-ku, Yokohama 232-0024, Japan
} 
the mainstay of treatment for advanced castration-sensitive prostate cancer. Despite the effective blocking of androgen biosynthesis and androgen receptor (AR) signalling, most of patients eventually progress and the disease ultimately becomes castration-resistant [5].

Apalutamide, a second generation nonsteroidal potent AR antagonist, selectively binds to the ligand-binding domain of AR with seven-tenfold greater affinity than the clinically approved antiandrogen, bicalutamide. In addition, in contrast to bicalutamide, apalutamide lacks significant AR agonist activity in preclinical models of CRPC and is unable to induce AR nuclear translocation and DNA binding in prostate cancer cells [6]. Apalutamide has a major metabolite JNJ-56142060, a pharmacologically active AR antagonist, with approximately threefold less potency than apalutamide.

In the previous phase $1 / 2$ study [7], apalutamide was safe and well-tolerated at 30-480 mg dose in non-Japanese castration-resistant prostate cancer (CRPC) patients. In the phase 2 part of that study [7], apalutamide at the $240 \mathrm{mg}$ daily dose was safe with promising efficacy [prostate-specific antigen (PSA) response] in patients with non-metastatic-CRPC (nmCRPC) and metastatic-CRPC (mCRPC). The present study was conducted to evaluate the safety, tolerability, pharmacokinetics (PK), and the antitumor effects of apalutamide after a single-dose $(240 \mathrm{mg})$ and multipledose administration ( $240 \mathrm{mg}$ once-daily) of apalutamide in Japanese men with mCRPC.

\section{Patients and methods}

\section{Patients}

Japanese men aged $\geq 20$ years with $\mathrm{mCRPC}$ who had histologically confirmed adenocarcinoma of the prostate without neuroendocrine differentiation or small cell features were included in this study. Inclusion criteria also involved maintained castrate levels of testosterone [ $<50 \mathrm{ng} /$ $\mathrm{dL}(1.72 \mathrm{nmol} / \mathrm{L})]$ within 4 weeks and serum PSA level of $\geq 2 \mathrm{ng} / \mathrm{mL}$ within 2 weeks before enrolment, which had risen on $\geq 2$ successive occasions, $\geq 1$ week apart. Patients who received first generation antiandrogen as part of an initial combined androgen blockade therapy or as second-line hormonal therapy for continuing disease progression, or who progressed after AR antagonists, 5- $\alpha$ reductase inhibitors, estrogens, and any other anticancer therapy, including chemotherapy given in the adjuvant/neoadjuvant setting were required to be off those medications for $\geq 4$ weeks before first dose administration.

The exclusion criteria were a history or current metastases in the brain or untreated spinal-cord compression, progressive epidural disease, prior treatment with the second generation antiandrogens (e.g., enzalutamide), or CYP17 inhibitors (e.g., abiraterone acetate, orteronel, galeterone, and systemic ketoconazole). Patients with a history of seizure or condition that may predispose to seizure, or use of radiopharmaceutical agents (e.g., strontium-89) or investigational immunotherapy (e.g., sipuleucel-T) within 12 weeks or any investigational non-immunologic agent within 4 weeks before the first dose of study agent were also excluded. Patients who used concurrent therapy with medications known to lower the seizure threshold and strong CYP3A4 inhibitors or inducers, herbal, and non-herbal products that could decrease serum PSA level, systemic (oral/IV/ IM) corticosteroids, or any other experimental treatment on another clinical trial within 4 weeks before the first dose of study agent were excluded.

\section{Study design and treatment}

In this phase 1, open-label, multi-centre study, the safety, tolerability, PK, and preliminary evidence of the antitumor effects of apalutamide ( $240 \mathrm{mg}$, once-daily orally) was evaluated in Japanese patients with mCRPC. This study comprised 3 periods: a screening period ( 28 days before study treatment), a treatment period (PK week and continuous daily dosing), and a post-treatment safety follow-up period (30 days after treatment discontinuation). Dose-limiting toxicity (DLT) evaluation period was defined as the PK week (1 week) and Cycle 1 (4 weeks) (Fig. 1).

In PK week, patients received a single oral dose of apalutamide $240 \mathrm{mg}$ on day 1 . After a week, patients were reassessed for safety to check treatment-emergent adverse events (TEAEs), serious TEAEs, DLT, Grade 3 toxicities, and other safety parameters on Cycle 1 day 1 (day 8) and these safety profiles were compared with those of the phase 1 global study. If no new safety signals, no difference in the frequency or the degree of an event was reported, the safety profile was acceptable. The patients were then started on continuous daily oral therapy of apalutamide $240 \mathrm{mg}$ until disease progression, unacceptable toxicity, withdrawal of consent, or death, whichever occurred first. If any DLT was reported, the treatment was stopped and restarted only after the toxicities were resolved to $\leq$ Grade 1 or baseline. After recovery, the dose was to be reduced accordingly (level 1: $180 \mathrm{mg} /$ day, level 2: $120 \mathrm{mg} /$ day) and restarted in accordance with the original schedule. Patients were to be withdrawn from the study if $>2$ dose-level reduction was required or if the treatment did not restart within 28 days after occurrence of toxicity in principle. Intra-patient dose escalation was not allowed.

The study protocol and amendments were approved by the local Institutional Review Board and the study was conducted in accordance with the ethical principles outlined in the Declaration of Helsinki. The study was consistent with the International Conference on Harmonization, Good 


\section{DLT evaluation period

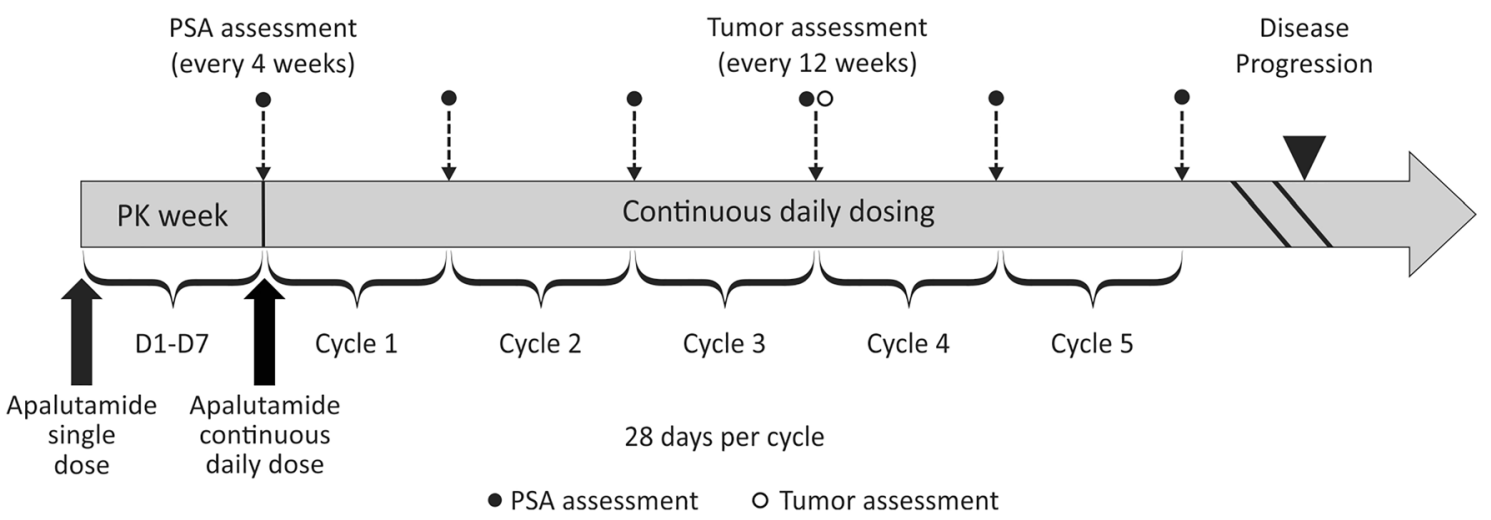

Fig. 1 Schematic overview of the study. $D 1-D 7$ day 1 to day 7, DLT dose-limiting toxicity, $P K$ pharmacokinetics, $P S A$ prostate-specific antigen

Clinical Practice guidelines, and applicable regulatory requirements. Written informed consent was obtained from all patients to participate in the study. The study was registered at ClincalTrials.gov: NCT02162836.

\section{Study evaluation}

\section{Safety and tolerability evaluation}

Safety assessments included results of TEAEs, clinical laboratory tests, vital signs (blood pressure, pulse rate, and body temperature) and Eastern Cooperative Oncology Group (ECOG) performance status, 12-lead electrocardiograms findings, and physical examination. All TEAEs were graded using the National Cancer Institute Common Terminology Criteria for Adverse Events version 4.0 (NCI CTCAE v4.0).

The tolerability of apalutamide $240 \mathrm{mg}$ in Japanese patients was evaluated based on the incidence of DLT (Online Resource 1). A study evaluation team (SET) was involved for final evaluation of toxicities based on DLT criteria, and for final decision on the safety and tolerability of apalutamide $(240 \mathrm{mg}$ ). DLTs were assessed during the PK week and Cycle 1 . Safety and tolerability of apalutamide $240 \mathrm{mg}$ in Japanese patients with mCRPC were confirmed, if $\leq 1 / 6$ patients experienced DLT. TEAEs of special interest (seizure, skin rash, hypothyroid, fall, and fracture) were also assessed.

\section{Pharmacokinetics}

Venous blood samples (approximately $3 \mathrm{~mL}$ each) for the determination of plasma concentrations of apalutamide and JNJ-56142060 (active metabolite) were collected and analyzed using a validated, specific, and sensitive liquid chromatography/mass spectrometry/mass spectrometry method. The plasma PK parameters for apalutamide, $C_{\max }$ (maximum observed plasma concentration), $t_{\max }$ (time to reach the maximum observed plasma concentration), $t_{1 / 2 \lambda}$ [elimination half-life associated with the terminal slope $(\lambda z)$ of the semi-logarithmic drug concentration-time curve, calculated as $0.693 / \lambda z$ ], and $\mathrm{AUC}_{0-24}$ (area under the plasma concentration-time curve from time $0-24 \mathrm{~h}$ postdose) were determined via non-compartmental analysis with Phoenix WinNonlin (Version 6.4). Parameters $\mathrm{AUC}_{\text {last }}$ [AUC from time 0 to time the last observed quantifiable concentration $\left.\left(C_{\text {last }}\right)\right]$ and $\mathrm{AUC}_{\infty}$ (AUC from time 0 to infinite time, calculated as the sum of $\mathrm{AUC}_{\text {last }}$ and $C_{\text {last } / \lambda z}$ ) were determined after a single oral administration (PK week). The parameters determined only after multiple oral administrations (Cycle 1 and later) were accumulation (Acc) index, effective half-life (EHL), percentage peak to trough fluctuation (PTF)\%, and $C_{\text {trough }}$ (trough plasma concentration just before dose). The following plasma $\mathrm{PK}$ parameters were determined for the metabolite JNJ-56142060: $C_{\text {max }}, t_{\max }, \mathrm{AUC}_{0-24}, C_{\text {trough }}$, Acc index, and metabolite-to-parent ratio (MPR).

\section{Antitumour efficacy assessment}

Assessment of antitumor activity of apalutamide was based on percentage change in serum PSA level from baseline reported at week 12 (or after treatment discontinuation, whichever occurred first). The maximal change was also reported for each patient using waterfall plots, at any time during the study. Serum PSA level was measured at screening visit, day 1 of PK week, day 1 of every cycle, and the end of treatment (EOT) visit by a central laboratory. Soft-tissue disease (measured by CT scans) and bone disease (evaluated radionuclide bone scans) were assessed at screening visit, 
and day 1 of every 3 cycles (e.g., Cycles 4, 7, 10, and so on), and the EOT visit.

\section{Criteria for disease progression}

Apalutamide was continued until both PSA progression and radiographic progression were documented, or until clinical progression such as a skeletal-related event, or until the treating physician decided to initiate a new systemic anticancer therapy. PSA progression was defined as PSA of $\geq 25 \%$ and $\geq 2 \mathrm{ng} / \mathrm{mL}$ above the nadir, which was confirmed by a second value obtained $\geq 3$ weeks later or defined as PSA of $\geq 25 \%$ and $\geq 2 \mathrm{ng} / \mathrm{mL}$ above baseline after week 12. Visible soft-tissue tumors [measurable (target) or non-measurable (non-target) lesions] were evaluated using RECIST Version 1.1. Radiographic progression was defined as soft-tissue progression evaluated by RECIST Version 1.1, which was confirmed on repeat imaging $\geq 6$ weeks later or by bone progression by Prostate Cancer Clinical Trials Working Group (PCWG) 2 [8].

Symptomatic clinical progression was defined by development of a skeletal-related event (pathologic fracture, spinal-cord compression, and need for surgery on bone or radiotherapy to bone) or progression of pain (decision was based on principal investigator's judgement; and as pain status was an exploratory endpoint, it was not checked on regular basis). The appearance of $\geq 2$ new lesions on day 1 of
Table 1 Demographic and baseline characteristics (alltreated population)

\begin{tabular}{|c|c|}
\hline & Apalutamide $240 \mathrm{mg}(N=6)$ \\
\hline Age (years), median (range) & $78.0(70-85)$ \\
\hline BMI $\left(\mathrm{kg} / \mathrm{m}^{2}\right)$, mean $(\mathrm{SD})$ & $22.04(0.973)$ \\
\hline Time from initial diagnosis to first dose (months), median (range) & $68.9(9.2-132.7)$ \\
\hline PSA at initial diagnosis (ng/mL), median (range) & $112.20(4.8-1169.3)$ \\
\hline \multicolumn{2}{|l|}{ Extent of disease at screening, $n(\%)$} \\
\hline Bone & $4(66.7)$ \\
\hline Bone only & $1(16.7)$ \\
\hline Soft tisssue & $5(83.3)$ \\
\hline Lymph node & $3(50.0)$ \\
\hline Prostate mass & $4(66.7)$ \\
\hline Other & $2(33.3)$ \\
\hline \multicolumn{2}{|l|}{ Tumor stage at initial diagnosis, $n(\%)$} \\
\hline $\mathrm{T} 2, \mathrm{~T} 2 \mathrm{a}, \mathrm{T} 2 \mathrm{~b}, \mathrm{~T} 2 \mathrm{c}$ & $3(50.0)$ \\
\hline T3, T3a, T3b & $2(33.3)$ \\
\hline $\mathrm{T} 4$ & $1(16.7)$ \\
\hline \multicolumn{2}{|l|}{ Metastasis stage at initial diagnosis, $n(\%)$} \\
\hline M0 & $4(66.7)$ \\
\hline M1, M1a, M1b, M1c & $2(33.3)$ \\
\hline \multicolumn{2}{|l|}{ ECOG performance status score at screening, $n(\%)$} \\
\hline 0 & $5(83.3)$ \\
\hline 1 & $1(16.7)$ \\
\hline \multicolumn{2}{|l|}{ Gleason score at initial diagnosis, $n(\%)$} \\
\hline$<7$ & $1(16.7)$ \\
\hline 7 & $1(16.7)$ \\
\hline$\geq 8$ & $4(66.7)$ \\
\hline \multicolumn{2}{|l|}{ Prior therapy, $n(\%)$} \\
\hline Hormonal therapy & $6(100.0)$ \\
\hline Chemotherapy & 0 \\
\hline Radiotherapy & $1(16.7)$ \\
\hline Surgery & $2(33.3)$ \\
\hline Prostate & $1(16.7)$ \\
\hline Orchiectomy & $1(16.7)$ \\
\hline Other & $2(33.3)$ \\
\hline
\end{tabular}

$B M I$ body mass index, ECOG Eastern Cooperative Oncology Group, PSA prostate-specific antigen, $S D$ standard deviation 
Cycle 4 visit with $\geq 2$ additional new lesions on confirmatory bone scan was considered as disease progression.

\section{Statistical methods}

Six patients were to be enrolled in the study to evaluate the tolerability of apalutamide at $240 \mathrm{mg}$. However, sample size was not based on statistical considerations. All-treated population set was defined as all patients who received $\geq 1$ dose of apalutamide, PK analysis population set was defined as all patients who received $\geq 1$ dose of apalutamide and had $\geq 1$ post-treatment sample collected for PK evaluation. All-treated population set was used for efficacy and safety analyses. Patients were excluded from the PK analysis if their data did not allow accurate assessment of the PK. Plasma concentrations and corresponding PK parameters for apalutamide and JNJ-56142060 were summarized using descriptive statistics.

Percentage change from baseline in serum PSA level at week 12 was summarized using descriptive statistics and presented graphically using waterfall plots. PSA and percentage change from baseline was also presented graphically using individual case plot. Time to PSA progression was summarized using descriptive statistics. The median time of PSA progression was estimated by Kaplan-Meier method.

\section{Results}

\section{Patients}

A total of 15 patients were screened and 6 patients of those were enrolled and treated with apalutamide at 4 sites in Japan. At the time of clinical data cutoff (15 March 2017), $5 / 6$ patients $(83.3 \%$ ) discontinued treatment and $1 / 6$ patient (16.7\%) was continuing the treatment. The treatment discontinuation was primarily due to disease progression $[3 / 6$ patients $(50.0 \%)]$, physician decision, and withdrawal of consent [1/6 patient (16.7\%) each].

Patient demographics and baseline characteristics are shown in Table 1. Overall, the median age of the patients was 78.0 (range 70-85) years; mean (SD) body mass index (BMI) was $22.04 \mathrm{~kg} / \mathrm{m}^{2}$ (0.973). All patients had an ECOG performance status of $0-1$. All patients received prior hormonal therapy and none of them received chemotherapy.

\section{Treatment compliance and extent of exposure}

Overall, median (range) of drug compliance was $98.3 \%$ (69.3-100.0\%). Of total, $3 / 6$ patients $(50 \%)$ achieved $100 \%$ compliance, $2 / 6$ patients (33.3\%) achieved $\geq 80$ to $\leq 100 \%$ compliance and 1 patient $(16.7 \%)$ achieved $<80 \%$ compliance. The median duration of study agent from day 1 Cycle 1 (except PK week) was 169.0 (range 74-869) days. At the time of clinical cutoff, all the 6 patients had received at least 3 cycles and 2 patients (33.3\%) received at least 30 cycles of study agent. The median average daily dose of study agent was 236.0 (range 170.3-240.0) mg/day.

\section{Safety}

\section{Adverse events}

All patients experienced at least one TEAE. The most frequently reported TEAEs ( $>1$ patient) were abdominal discomfort, nasopharyngitis, dysgeusia, rash, and hot flush [2/6 patients (33.3\%) each] (Table 2). The drug-related TEAEs reported in $>1$ patient were abdominal discomfort, dysgeusia, and hot flush [2/6 patients (33.3\%) each]. One patient $(16.7 \%)$ reported serious TEAE (Grade 3 spinal-cord compression) and, therefore, discontinued the study agent. A total of $2 / 6$ patients $(33.3 \%)$ reported Grade 3 TEAEs [spinal-cord compression and renal disorder, $1 / 6$ patients $(16.7 \%)$ each]. However, at data cutoff, renal disorder was resolved, while the event of spinal-cord

Table 2 Overview of treatment-emergent adverse events through week 24 (all-treated population)

\begin{tabular}{ll}
\hline & $\begin{array}{l}\text { Apalutamide } \\
240 \mathrm{mg} \\
(N=6) \\
N(\%)\end{array}$ \\
\hline Patients with $\geq 1$ TEAEs & $6(100)$ \\
Serious TEAEs & $1(16.7)$ \\
Grade 3 or higher TEAEs & $2(33.3)$ \\
Spinal-cord compression & $1(16.7)$ \\
Renal disorder & $1(16.7)$ \\
TEAEs leading to discontinuation of study agent & $1(16.7)$ \\
Dose-limiting toxicity & 0 \\
Death & 0 \\
TEAEs $\geq 2$ patients & \\
Abdominal discomfort & $2(33.3)$ \\
Nasopharyngitis & $2(33.3)$ \\
Dysgeusia & $2(33.3)$ \\
Rash & $2(33.3)$ \\
Hot flush & $2(33.3)$ \\
TEAEs of special interest & \\
Rash & $2(33.3)$ \\
Rash generalized & $1(16.7)$ \\
Rib fracture & $1(16.7)$ \\
\hline
\end{tabular}

TEAEs treatment-emergent adverse events

${ }^{a}$ TEAEs of special interest includes seizure, skin rash, hypothyroid, fall, and fracture 
compression was resolving. No death and no DLTs were reported. Dose interruption was reported in $2 / 6$ patients (33.3\%) due to TEAEs [Grade 2 rash generalized and Grade 1 toxic skin eruption, $1 / 6$ patients $(16.7 \%)$ each]. Three patients $(50.0 \%)$ were reported with TEAEs of special interest (rash in 2 patients and rib fracture in 1 patient). The Grade 1 rash of the special interest TEAE resolved in both the patients without any action on dose of the study agent; however, it reoccurred in one patient as Grade 2 rash generalized on day 55. The event of rash generalized resolved on day 74 and reoccurred on day 83 with similar grade. It was reported to be resolving after dose interruption at time of clinical cutoff. Grade 1 rib fracture was observed in $1 / 6$ patient (16.7\%), no action on dose of the study agent was taken and the event was not recovered at clinical cutoff date. No seizure, hypothyroid, or fall was reported (Table 2).

\section{Clinical laboratory evaluation}

The Grade 3 hematology laboratory abnormalities were lymphocyte count decreased and lymphocyte count increased [1/6 patient (16.7\%) each].

\section{Pharmacokinetic assessment}

PK analysis was performed in all the six patients; however, one patient was excluded from the descriptive statistical analysis due to vomiting after the first dose. Nonetheless, after multiple doses, parameters not affected by vomiting were included. A steady state of apalutamide was reached approximately by day 22 of Cycle 1 (week 4 ), as indicated by the mean trough concentration-time profile (data not shown). The median $t_{\max }$ of apalutamide was reached at $1.58 \mathrm{~h}$ after single-dose administration and $1.44 \mathrm{~h}$ after

Table 3 Summary of PK parameters of apalutamide and its metabolite JNJ-56142060 in plasma (PK analysis population)

\begin{tabular}{|c|c|c|c|c|}
\hline & \multicolumn{2}{|l|}{ Apalutamide $240 \mathrm{mg}$} & \multicolumn{2}{|l|}{ JNJ-56142060 } \\
\hline & PK week day $1(N=5)$ & Cycle 1 day $22(N=6)$ & PK week day $1(N=5)$ & Cycle 1 day $22(N=6)$ \\
\hline \multicolumn{5}{|l|}{$C_{\max }(\mu \mathrm{g} / \mathrm{mL})$} \\
\hline Mean (SD) & $3.88(0.793)$ & $7.57(1.19)$ & $0.366(0.0751)$ & $7.11(0.551)$ \\
\hline $\mathrm{CV} \%$ & 20.4 & 15.7 & 20.5 & 7.80 \\
\hline \multicolumn{5}{|l|}{$t_{\max }(\mathrm{h})$} \\
\hline Median & 1.58 & 1.44 & 168 & 3.68 \\
\hline Range & $(1.00-2.05)$ & $(0.950-4.00)$ & $(95.5-168)$ & $(0.00-23.8)$ \\
\hline \multicolumn{5}{|c|}{$\mathrm{AUC}_{0-24}(\mathrm{~h} \mu \mathrm{g} / \mathrm{mL})$} \\
\hline Mean (SD) & $33.6(4.78)$ & $122(17.5)$ & $2.69(0.307)$ & $150(15.6)$ \\
\hline $\mathrm{CV} \%$ & 14.2 & 14.3 & 11.4 & 10.4 \\
\hline \multicolumn{5}{|c|}{$\mathrm{AUC}_{\text {last }}(\mathrm{h} \mu \mathrm{g} / \mathrm{mL})$} \\
\hline Mean (SD) & $116(14.1)$ & - & $45.4(8.59)$ & - \\
\hline $\mathrm{CV} \%$ & 12.1 & - & 18.9 & - \\
\hline \multicolumn{5}{|l|}{ Acc index } \\
\hline Mean (SD) & - & $3.55(0.139)^{\mathrm{a}}$ & - & $57.3(6.41)^{\mathrm{a}}$ \\
\hline $\mathrm{CV} \%$ & - & 3.90 & - & 11.2 \\
\hline \multicolumn{5}{|l|}{ EHL (h) } \\
\hline Mean (SD) & - & $50.2(2.34)^{\mathrm{a}}$ & - & - \\
\hline $\mathrm{CV} \%$ & - & 4.70 & - & - \\
\hline \multicolumn{5}{|l|}{ PTF\% (\%) } \\
\hline Mean (SD) & - & $177(23.5)$ & - & - \\
\hline $\mathrm{CV} \%$ & - & 13.2 & - & - \\
\hline \multicolumn{5}{|l|}{ MPR for $C_{\max }$} \\
\hline Mean (SD) & - & - & $0.0987(0.0314)$ & $0.951(0.104)$ \\
\hline $\mathrm{CV} \%$ & - & - & 31.8 & 10.9 \\
\hline \multicolumn{5}{|c|}{ MPR for $\mathrm{AUC}_{0-24}$} \\
\hline Mean (SD) & - & - & $0.0814(0.0159)$ & $1.24(0.159)$ \\
\hline CV\% & - & - & 19.6 & 12.8 \\
\hline
\end{tabular}

Acc accumulation, $C V$ coefficient of variation, $E H L$ effective half-life, $M P R$ metabolite-to-parent ratio, $P K$ pharmacokinetics, $P T F$ peak to trough fluctuation, $S D$ standard deviation

${ }^{\mathrm{a}} N=5$ patients 
multiple-dose administration. The mean $C_{\max }$ of apalutamide was approximately twofold higher after multipledose administration $(7.57 \mu \mathrm{g} / \mathrm{mL}$ at Cycle 1 day 22) compared with single-dose administration $(3.88 \mu \mathrm{g} / \mathrm{mL}$ at PK week day 1). On Cycle 1, day 22, mean Acc index based on $\mathrm{AUC}_{0-24}$ of apalutamide was 3.55. After single-dose administration, individual $t_{1 / 2 \lambda}$ of apalutamide ranged from 110 to $231 \mathrm{~h}$; however, the data should be cautiously interpreted owing to the short sampling period (up to $168 \mathrm{~h}$ ). After multiple-dose administration, mean EHL was $50.2 \mathrm{~h}$ and mean PTF\% was $177 \%$ for apalutamide. A steady state of JNJ-56142060 was reached approximately by day 1 of Cycle 2 (week 5) (data not shown). The mean $C_{\max }$ of JNJ-56142060 was approximately 19-fold higher after multiple-dose administration $(7.11 \mu \mathrm{g} / \mathrm{mL}$ at Cycle 1 day 22) compared with single-dose administration $(0.366 \mu \mathrm{g} /$ $\mathrm{mL}$ at PK week day 1). Mean Acc index based on $\mathrm{AUC}_{0-24}$ of JNJ-561402060 was 57.3. Mean MPR after single-dose administration and multiple-dose administration for AUC $0-24$ was 0.0814 and 1.24 , respectively (Table 3 ).

\section{Efficacy assessment}

The median serum PSA level decreased from 54.42 (range $8.92-310.11) \mathrm{ng} / \mathrm{mL}$ at baseline to 11.70 (range 0.37-47.74) $\mathrm{ng} / \mathrm{mL}$ at week 12 . Most of the patients [4/6 patients $(66.7 \%)]$ achieved at least $50 \%$ reduction from baseline in serum PSA level at week 12 and a few patients could also achieve $90 \%$ reduction [2/6 patients (33.3\%)] (Fig. 2). The median maximum reduction of percentage change from baseline in serum PSA level during treatment was $-88.3 \%$ (range -41.6 to $-99.8 \%$ ) (Table 4 ).

At clinical cutoff, $4 / 6$ patients showed progression of disease [PSA progression and radiographic progression: $2 / 6$ patients (33.3\%), clinical progression: $1 / 6$ patients (16.7\%), disease progression (PSA was reported to be $>40$ and no pain progression was observed) confirmed by the investigator: $1 / 6$ patients $(16.7 \%)$ ]. One patient with radiographic disease progression had bone metastases and the other patient had metastatic progression of prostate mass at periaortic lymph node and both sides of internal iliac artery lymph node. The patient who did not meet predefined progression of disease criteria showed increase in serum PSA level with no pain progression.

\section{Discussion}

This open-label study assessed the safety, tolerability, PK, and antitumor effects of apalutamide after single and multiple doses in Japanese men with mCRPC. Apalutamide administered at $240 \mathrm{mg}$ once-daily dose showed manageable toxicity and overall TEAEs were consistent with the safety profile reported in global phase 1 studies of apalutamide [9-12]. No deaths, drug-related serious TEAEs, any Grade 4 or higher TEAEs, DLTs or seizure were reported in this study. Two patients required dose interruptions due to TEAEs.

After single-dose administration of apalutamide $240 \mathrm{mg}$, mean $C_{\max }$ and $\mathrm{AUC}_{0-24}(3.88 \mu \mathrm{g} / \mathrm{mL}$ and $33.6 \mu \mathrm{g} \mathrm{h} / \mathrm{mL}$, respectively) in Japanese patients with $\mathrm{mCRPC}$ were higher than $C_{\text {max }}$ and $\mathrm{AUC}_{0-24}(2.97 \mu \mathrm{g} / \mathrm{mL}$ and $21.9 \mu \mathrm{g} \mathrm{h} / \mathrm{mL}$, respectively; unpublished data) observed in non-Japanese patients with mCRPC $(N=3)$. However, similar mean $C_{\max }$ ( $7.57 \mathrm{vs} 7.55 \mu \mathrm{g} / \mathrm{mL}$ ) and $\mathrm{AUC}_{0-24}(122 \mathrm{vs} 127 \mu \mathrm{g} \mathrm{h} / \mathrm{mL}$ ) were observed in Japanese vs non-Japanese patients with mCRPC, after multiple-dose administration of apalutamide $240 \mathrm{mg}$ [9]. Moreover, mean MPR for $\mathrm{AUC}_{0-24}$ in Japanese and non-Japanese patients with mCRPC were also comparable (1.24 vs 1.09; unpublished data).

The efficacy results of this phase 1 study demonstrated durable decline in PSA with apalutamide $240 \mathrm{mg}$ once-daily dose, suggesting that the drug was effective and showed comparable trend with the result of global phase $1 / 2$ study (NCT01171898) [7, 12]. In this study, a $\geq 50 \%$ reduction in serum PSA level from baseline was confirmed in $4 / 6$ patients (66.7\%) at week 12. Apalutamide $240 \mathrm{mg}$ once-daily dose was approved in US [13] and Japan [14] for nmCRPC following positive results from the phase 3 SPARTAN study, where apalutamide confirmed significant improvements in metastasis-free survival $(P<0.001)$ and progression-free

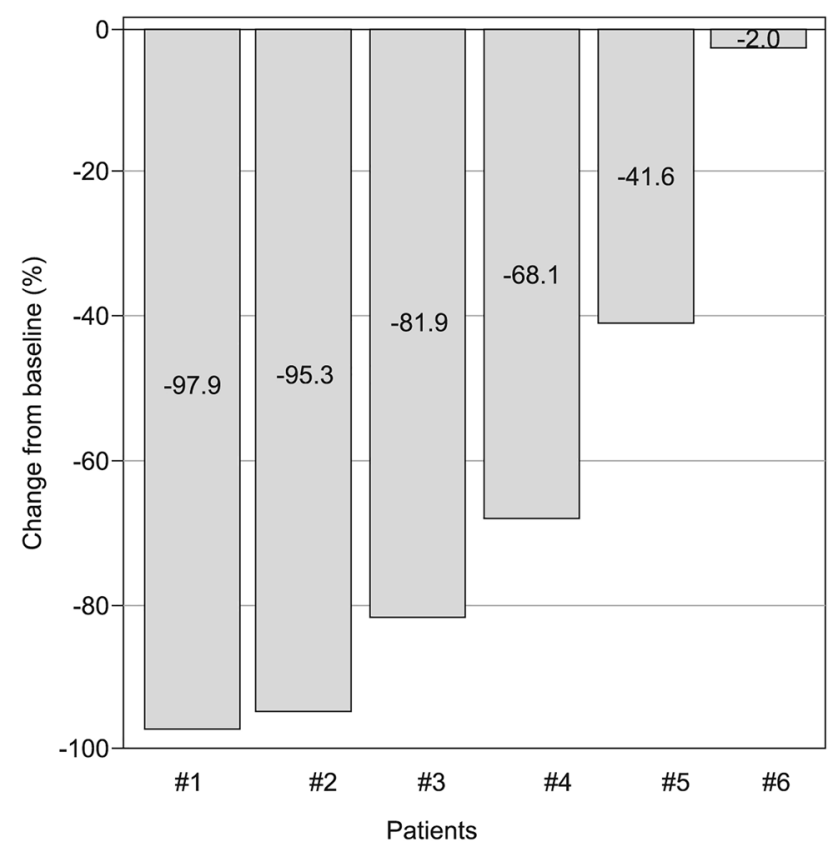

Fig. 2 Waterfall plots of prostate-specific antigen percent change from baseline at week 12 (all-treated population) 
Table 4 Summary of prostatespecific antigen assessment (all-treated population)
Apalutamide $240 \mathrm{mg}(N=6)$

\begin{tabular}{ll}
\hline PSA (ng/mL) & \\
Baseline, median (range) & $54.420(8.92-310.11)$ \\
12 weeks, median (range) & $11.700(0.37-47.74)$ \\
Percent change from baseline at 12 weeks, median (range) & $-75.04(-97.9$ to -2.0$)$ \\
Maximum reduction from baseline, median (range) & $-29.630(-295.45$ to -3.77$)$ \\
Maximum reduction of percent change from baseline, median (range) & $-88.26(-99.8$ to -41.6$)$ \\
PSA progression & \\
Number of events (\%) & $2(33.3)$ \\
Number of censored (\%) & $4(66.7)$ \\
Time to event (months) & \\
$\quad$ Median (95\% CI) & $27.89(5.82, \mathrm{NE})$ \\
\hline
\end{tabular}

$C I$ confidence interval, $N E$ not evaluable, $P S A$ prostate-specific antigen survival $(P<0.001)$ as compared to placebo [15]. Apalutamide also showed a very good risk-benefit balance in phase 3 TITAN study involving Japanese patients with metastatic castration-sensitive prostate cancer (mCSPC), where apalutamide confirmed significant improvements in radiographic progression-free survival $(P<0.001)$ and overall survival $(P<0.005)$ as compared to placebo [16].

The small sample size of the study restrains reliable demonstration of the efficacy. However, the small sample size is commonly observed in early development studies and is sufficient to allow clinical judgment of safety and tolerability, and assessment of PK profile. Furthermore, as this was a single-dose study, it was difficult to determine dose proportionality.

In conclusion, apalutamide $240 \mathrm{mg}$ was well-tolerated without any DLT or any new safety signals with a favourable efficacy in Japanese mCRPC patients. In addition, the pharmacokinetic profile of apalutamide $240 \mathrm{mg}$ once-daily in Japanese mCRPC patients was consistent with the established profile in the non-Japanese population. Thus, apalutamide $240 \mathrm{mg}$ (once-daily, orally) was ascertained to be an adequate dosage regimen in Japanese mCRPC patients.

Acknowledgements Rukhsar Wasta, M Pharm and Varkha Agrawal, $\mathrm{PhD}$ provided writing assistance and Sangita Patil, PhD, CMPP (all from SIRO Clinpharm Pvt. Ltd., India) provided additional editorial support for this manuscript. Authors also thank the study participants, without whom this study would never have been accomplished, and the investigators for their participation in this study. Mr. Hidehisa Noguchi (ex-employee of Janssen pharmaceutical KK, Japan) provided substantial contribution at the manuscript development stage.

Author contributions KI, YI, and RO contributed to conception and design of the study. YI was involved in data analysis. All authors were involved in data interpretation, development, and critical revision of the manuscript for intellectual content. All authors had full access to all the data in the study and take responsibility for the integrity of the data and the accuracy of the data analysis. All authors meet ICMJE criteria and all those who fulfilled those criteria are listed as authors. All authors provided direction and comments on the manuscript, made the final decision about where to publish these data, and approved submission to the journal.

Funding This work was supported by Janssen Pharmaceutical K.K., Tokyo, Japan.

\section{Compliance with ethical standards}

Conflict of interest Dr. Hiroji Uemura received grants from Janssen Pharmaceutical K.K.(during the conduct of the study) and from Bayer Yakuhin, Limited, Takeda Pharmaceutical Company Limited, AstraZeneca K.K., TAIHO Pharmaceutical Company Limited, Astellas Pharma Inc. and Pfizer Inc (outside the research work, lecture, or subsidies). Dr. Katsuyoshi Hashine received grants from Janssen Pharmaceutical (during the conduct of the study) and from Takeda Pharmaceutical (subsidies). Dr. Tomohiro Tsuchiya and Dr. Akito Yamaguchi received grants from Janssen Pharmaceutical K.K. during the conduct of the study. Dr. Keiichiro Imanaka, Ms. Yuki Iwaki, and Mr. Ryo Oyama are employees of Janssen Pharmaceutical K.K.

Open Access This article is distributed under the terms of the Creative Commons Attribution 4.0 International License (http://creativeco mmons.org/licenses/by/4.0/), which permits unrestricted use, distribution, and reproduction in any medium, provided you give appropriate credit to the original author(s) and the source, provide a link to the Creative Commons license, and indicate if changes were made.

\section{References}

1. Bray F, Jerlay J, Soerjomataram I et al (2018) Global cancer statistics 2018: GLOBOCAN estimates of incidence and mortaility worldwide for 36 caancers in 185 countries. CA Cancer J Clin 68(6):394-424

2. Foundation for promotion of cancer research (2014) Cancer statistics in Japan-2014. Available via DIALOG. https://ganjoho.jp/ data/reg_stat/statistics/brochure/2014/cancer_statistics_2014_ fig_E.pdf. Accessed Nov 2018

3. Hori M, Matsuda T, Shibata A et al (2015) Cancer incidence and incidence rates in Japan in 2009: a study of 32 population-based cancer registries for the Monitoring of Cancer Incidence in Japan (MCIJ) project. Jpn J Clin Oncol 45(9):884-891 
4. Matsubara N, Mukai H, Hosono A et al (2017) Phase 1 study of darolutamide (ODM-201): a new-generation androgen receptor antagonist, in Japanese patients with metastatic castration-resistant prostate cancer. Cancer Chemother Pharmacol 80(6):1063-1072

5. Chong JT, Oh WK, Liaw BC (2018) Profile of apalutamide in the treatment of metastatic castration-resistant prostate cancer: evidence to date. OncoTargets Ther 11:2141-2147

6. Clegg NJ, Wongvipat J, Joseph JD et al (2012) ARN-509: a novel anti-androgen for prostate cancer treatment. Cancer Res 72(6):1494-1503

7. Smith MR, Antonarakis ES, Ryan CJ et al (2016) Phase 2 study of the safety and antitumor activity of apalutamide (ARN-509), a potent androgen receptor antagonist, in the digh-risk nonmetastatic castration-resistant prostate cancer cohort. Eur Urol 70(6):963-970

8. Scher HI, Halabi S, Tannock I et al (2008) Design and end points of clinical trials for patients with progressive prostate cancer and castrate levels of testosterone: recommendations of the prostate cancer clinical trials working group. J Clin Oncol 26(7):1148-1159

9. Rathkopf DE, Morris MJ, Fox JJ et al (2013) Phase I study of ARN-509, a novel antiandrogen, in the treatment of castrationresistant prostate cancer. J Clin Oncol 31(28):3525-3530

10. Rathkopf DE, Slovin SF, Morris MJ et al (2017) Targeting reciprocal feedback inhibition: Apalutamide and everolimus in patients with metastatic castration-resistant prostate cancer (mCRPC). J Clin Oncol 35(6_suppl):204
11. Posadas EM, Chi KN, Wit RD et al (2017) Phase Ib study of apalutamide (APA) with abiraterone acetate (AA) and prednisone $(\mathrm{P})$ in patients (pts) with metastatic castration-resistant prostate cancer (mCRPC): update on safety and efficacy. J Clin Oncol 35(6_suppl): 173

12. Rathkopf DE, Smith MR, Ryan CJ et al (2017) Androgen receptor mutations in patients with castration-resistant prostate cancer treated with apalutamide. Ann Oncol 28(9):2264-2271

13. FDA approves apalutamide for non-metastatic castration-resistant prostate cancer. Available via DIALOG. https://www.fda.gov/ Drugs/InformationOnDrugs/ApprovedDrugs/ucm596796.htm. Accessed Oct 2018

14. List of approved products in Pharmaceuticals and Medical Devices Agency (PMDA). https://www.pmda.go.jp/files/00022 9856.pdf\#page $=8$. Accessed June 2019

15. Smith MR, Saad F, Chowdhury S et al (2018) Apalutamide treatment and metastasis-free survival in prostate cancer. N Engl J Med 378(15):1408-1418

16. Chi KN, Agarwal N, Bjartell A et al (2019) Apalutamide for metastatic, castration-sensitive prostate cancer. N Engl J Med. https:// doi.org/10.1056/NEJMoa1903307

Publisher's Note Springer Nature remains neutral with regard to jurisdictional claims in published maps and institutional affiliations. 